\title{
Análisis y Plan Quirúrgico de Deformidades en Tobillo y Retropié del Adulto
}

\section{Analysis and Surgical Planning in Ankle and Hindfoot Adult Deformities}

\author{
Mario López Morales ${ }^{1}$ Pablo Wagner Hitschfeld ${ }^{1,2}$ \\ ${ }^{1}$ Departamento de Traumatología y Ortopedia, Clínica Alemana - \\ Universidad del Desarrollo, Santiago, Chile \\ 2 Departamento de Traumatología y Ortopedia, Hospital Militar de \\ Santiago - Universidad de los Andes, Santiago, Chile \\ Rev Chil Ortop Traumatol 2020;61:28-35.
}

Address for correspondence Mario López Morales, Escandinavia 432 dpto 301, Las Condes, Región Metropolitana, Chile

(e-mail: malopez3@uc.cl).

\section{Resumen \\ Palabras claves \\ - alineamiento de extremidades \\ - extremidad inferior \\ - osteotomía \\ - retropié \\ - tobillo}

La corrección de deformidades en extremidades inferiores del adulto sigue siendo un capítulo desafiante en ortopedia y traumatología. El conocimiento del alineamiento normal de las extremidades inferiores y su comportamiento son fundamentales para una adecuada planificación quirúrgica y éxito del tratamiento, especialmente en tobillo y retropié. El objetivo de esta revisión, es conocer los principios fundamentales de la corrección de deformidades, orientar en que factores fijarse al momento de corregir y poder dar una guía de cómo planificar la cirugía, particularmente en deformidades de tobillo y retropié.

Nivel de evidencia: Nivel V.

Adult lower limb deformity corrections remain a challenging chapter in orthopedic surgery. The knowledge of the normal lower limb alignment and their behavior is essential for a proper surgical planning and treatment success, especially on foot and ankle surgery. The objective of this review is to show the main principles of deformity correction, to guide the factors to consider when correcting and to provide a surgical planning guide, particularly in the ankle and hind foot deformities.

\section{Introducción}

La deformidad se puede definir como cualquier desviación de la anatomía normal del hueso o articulación. ${ }^{1}$ Una deformidad se puede describir en relación a su plano coronal (frontal), sagital (lateral) y axial (transversal). Alternativamente, la deformidad se puede describir como traslación a lo largo y/o rotación alrededor de los ejes x, y, z, describiéndose seis grados de libertad.

Las causas de deformidad varían según la edad del paciente. En los niños la causa suele ser congénita (por ejemplo, hemimelia fibular, deficiencia focal femoral proximal). En adolescentes la deformidad puede surgir de traumas o patologías que afectan la placa de crecimiento, como infecciones, enfermedad de Blount, aclasia diafisaria y raquitismo. En los adultos, la mayoría de los casos son secundarios a no unión o mal unión de fracturas o enfermedades metabólicas como Paget, dejando secuelas devastadoras para la calidad de vida del paciente.

Una deformidad en la extremidad inferior tiende a cambiar la transferencia de carga de una articulación a otra. Eso tiene diferentes consecuencias, como alteración de la marcha, que received

April 24, 2019

accepted

October 11, 2019
DOI https://doi.org/

10.1055/s-0039-3400508. ISSN $0716-4548$.
Copyright (e 2020 by Thieme Revinter

Publicações Ltda, Rio de Janeiro, Brazil
License terms

c) $(1) \$$ 
lleva a fatiga muscular, sobrecarga de articulaciones y desgaste precoz del cartílago. Por ejemplo, una desviación en el plano coronal de la tibia se compensa a través de la articulación tibiotalar y subtalar, cambiando su área de contacto, sometiendo al cartílago a un aumento de presión focal, llevando a sinovitis y posterior artrosis secundaria.

El objetivo de esta revisión, es conocer los principios fundamentales de la corrección de deformidades, orientar en qué factores fijarse al momento de corregir y poder dar una guía de cómo planificar la cirugía, particularmente en deformidades de tobillo y retropié.

\section{Principios Básicos}

El conocimiento de los principios básicos de deformidad de las extremidades inferiores es imprescindible al momento de llevar a cabo la planificación quirúrgica. Esos principios proporcionan puntos de referencia precisos para producir resultados quirúrgicos predecibles.

Los parámetros normales se definen a partir de una teleradiografía de extremidades inferiores en proyección anteroposterior y lateral. ${ }^{2}$ En ella se define un eje mecánico (o eje de carga) y un eje anatómico (línea diafisiaria).

\section{Alineamiento en el Plano Frontal de la Extremidad Inferior}

El eje mecánico de la extremidad en el plano frontal se define como una línea directa que conecta el centro de la cabeza femoral con el centro del tobillo (-Figura 1). En personas normales, el eje mecánico pasa ligeramente medial al centro de la rodilla (considerándose normal que esa línea pase $\mathrm{a} \pm 4 \mathrm{~mm}$ del centro de ésta). ${ }^{3}$ Cuando el eje mecánico no pasa por el centro de la rodilla, se dice que la extremidad tiene una desviación del eje mecánico (MAD, "mechanical axis deviation"); cuando éste pasa medial se habla que el paciente tiene un "varo" y cuando pasa lateral un "valgo."

El eje anatómico en cambio, es una línea que sigue la diáfisis de los huesos largos. Ella puede ser recta o curva. En el fémur, la diferencia entre el eje anatómico y mecánico es de aproximadamente $7^{\circ}$ (rango, 5-9 $\left.{ }^{\circ}\right) .^{3}$ En la tibia, ambos ejes tienden a coincidir ( - Figura $\mathbf{1}$ ).

Ahora bien, la congruencia de la línea articular también afecta la carga de la extremidad. El ángulo de convergencia de la línea articular (JLCA, "joint line convergence angle") se forma por la línea articular distal del hueso proximal y la línea articular proximal del hueso distal. Se considera normal $\pm 2^{\circ}{ }^{4}$ Si es mayor a ese valor, hay que considerar que la línea de congruencia articular está contribuyendo a la deformidad de la extremidad.

\section{Alineamiento en el Plano Sagital de la Extremidad Inferior}

Al igual que el plano frontal, el sagital también tiene un eje mecánico y anatómico. El mecánico tiende a pasar ligeramente anterior al punto bisagra de la rodilla (intersección de la línea de Blumensaat y la cortical posterior, - Figura 2). ${ }^{3}$ Se considera normal que ese eje pase ligeramente anterior al punto bisagra y ligeramente posterior a los confines anteriores del fémur distal,

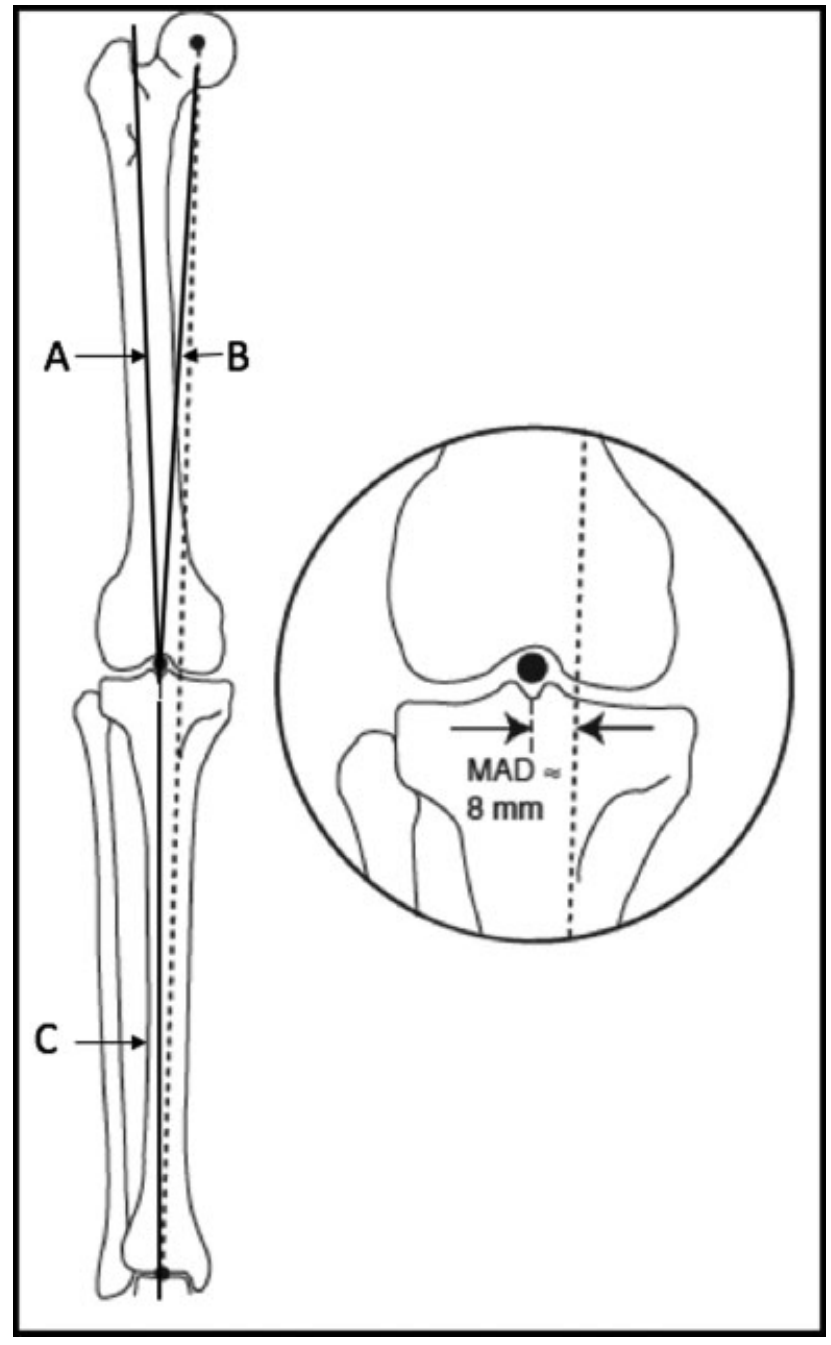

Fig. 1 (A) Eje anatómico fémur; (B) Eje mecánico fémur; (C) Eje anatómico y mecánico de fémur; Línea punteada, eje mecánico de extremidad. Lamm B, Paley D. Clin Podiatr Med Surg 21 (2004).

porque al ser así la rodilla puede "bloquearse" cuando la extremidad está totalmente extendida, permitiendo que el cuádriceps descanse durante periodos largos de bipedestación. Si no es así, al tener un flexo fijo, el cuádriceps tiene que estar activado en todo momento, resultando en fatiga, dolor patelofemoral y anormalidad de la marcha.

En el plano sagital, usamos el "verdadero" eje mecánico de la extremidad (centro de la cabeza femoral al centro del tobillo) para analizar el MAD. Sin embargo, usamos el eje mecánico "modificado" para trazar los ángulos articulares. ${ }^{5}$ Cuando dibujamos el eje modificado no se usa el centro del fémur distal o tibia proximal. Para identificar eje modificado femoral, se traza una línea que conecta el límite anterior y posterior de la línea fisiaria femoral y se intersecta en la unión del tercio anterior con los dos tercios posteriores, luego partiendo desde este punto hacia el centro de la cabeza femoral. Para identificar el eje modificado tibial, se traza una línea que conecta el borde anterior y posterior del platillo tibial y se marca un punto en la unión del quinto anterior con los cuatro quintos posteriores, luego se traza una línea que conecta las puntas anterior y posterior de la 


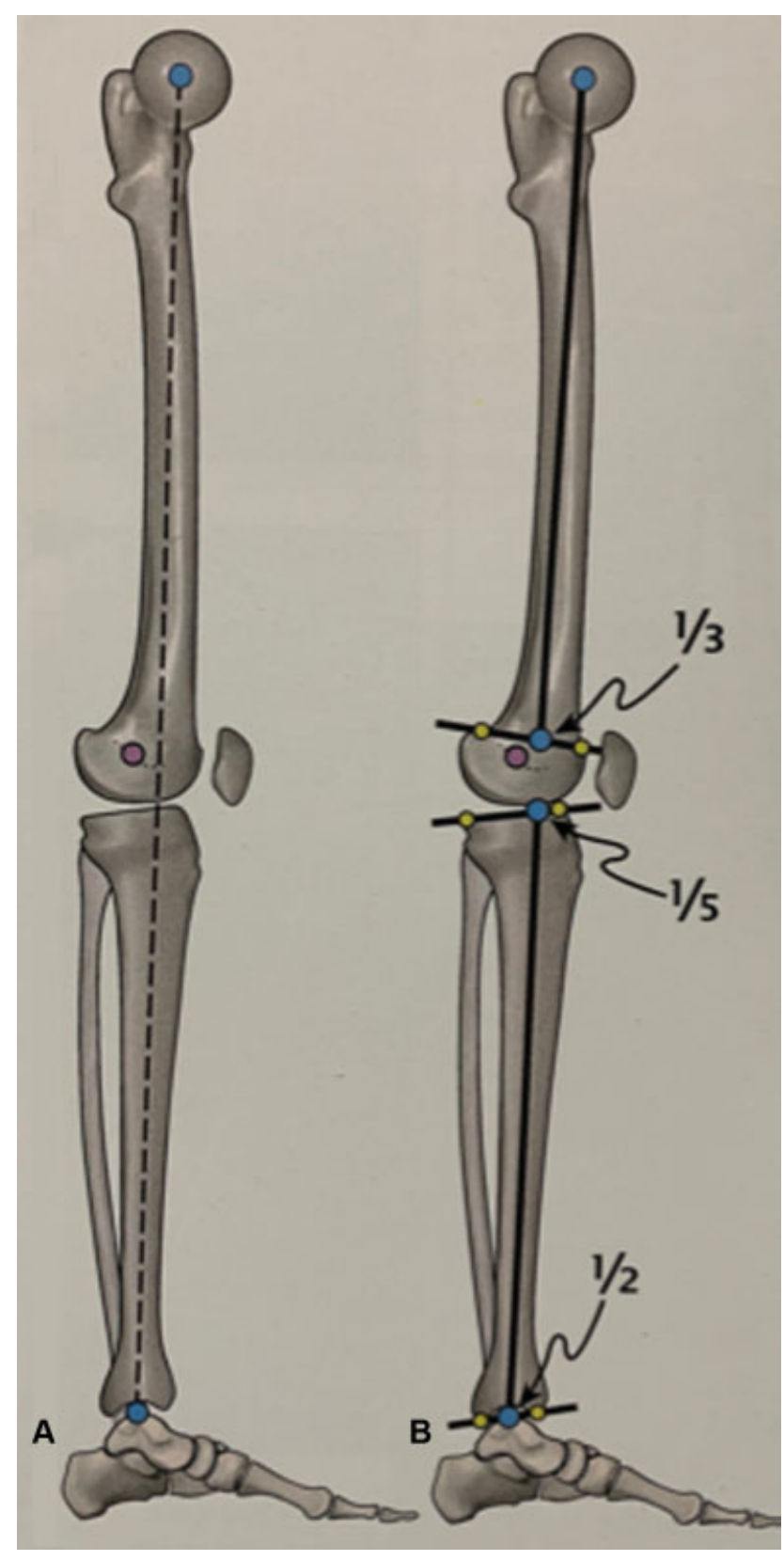

Fig. 2 (A) El "verdadero" eje mecánico de la extremidad; (B) eje mecánico "modificado" para analizar los ángulos de las articulaciones. The art of limb alignment. Shawn C. standard. tibia distal y se marca punto en el medio de ella. A continuación, se une el punto marcado en el platillo y en la tibia distal (-Figura 2).

Al analizar el plano sagital, se debe evaluar tanto el alineamiento óseo como los tejidos blandos, que pueden estar contribuyendo a la deformidad.

\section{Alineamiento del Tobillo y Retropié}

La articulación tibiotalar, o articulación del tobillo, es diferente a la articulación de la rodilla. La forma de la articulación tibiotalar se ve cuadrada en AP, circular en lateral; el eje anatómico de la tibia intersecta justo medial al punto medio del talo en el plano frontal e intersecta en el proceso lateral del talo (centro de rotación del tobillo) en el plano sagital. Dentro de los ángulos importantes a medir, en el plano AP está el ángulo plafond - maleolar $\left(9^{\circ} \pm 4\right)$ y talocrural $\left(82^{\circ} \pm 3,6\right)$; en el plano lateral el ángulo plantígrado $\left(90^{\circ}\right)$ y el aADTA ("anatomical anterior distal tibial angle," $\left.80^{\circ}\right)^{6}$ (-Figura 3 ).

Respecto al talón (calcáneo), en el plano frontal su eje es paralelo al eje anatómico de la tibia y su centro se encuentra aproximadamente a 5-10 $\mathrm{mm}$ lateral a él. En el plano sagital, el calcáneo está inclinado en $20-30^{\circ} .6$

\section{Reglas de la Osteotomía}

Para entender las reglas de osteotomía es necesario entender 2 conceptos básicos: el CORA y el ACA descritos por Paley y col. $^{3,7}$ El CORA (de su sigla en inglés "center of rotation of angulation), es el sitio donde se encuentra la deformidad. El ACA ("axis of correction of angulation") es el eje en el cual se va a corregir la deformidad. Actualmente, el término CORA fue reemplazo por "ápex de la deformidad" y el ACA por "hinge" o bisagra de corrección.

Primera regla: Si la osteotomía y el ápex de deformidad están en el mismo lugar, el hueso se va a realinear si el ángulo de corrección es igual al ápex, sin necesidad de trasladar el segmento.

Segunda regla: Si la bisagra pasa a través del ápex de deformidad, pero la osteotomía se hace a un nivel diferente, el eje del hueso se va a realinear si se cambia la angulación y la traslación de la osteotomía.

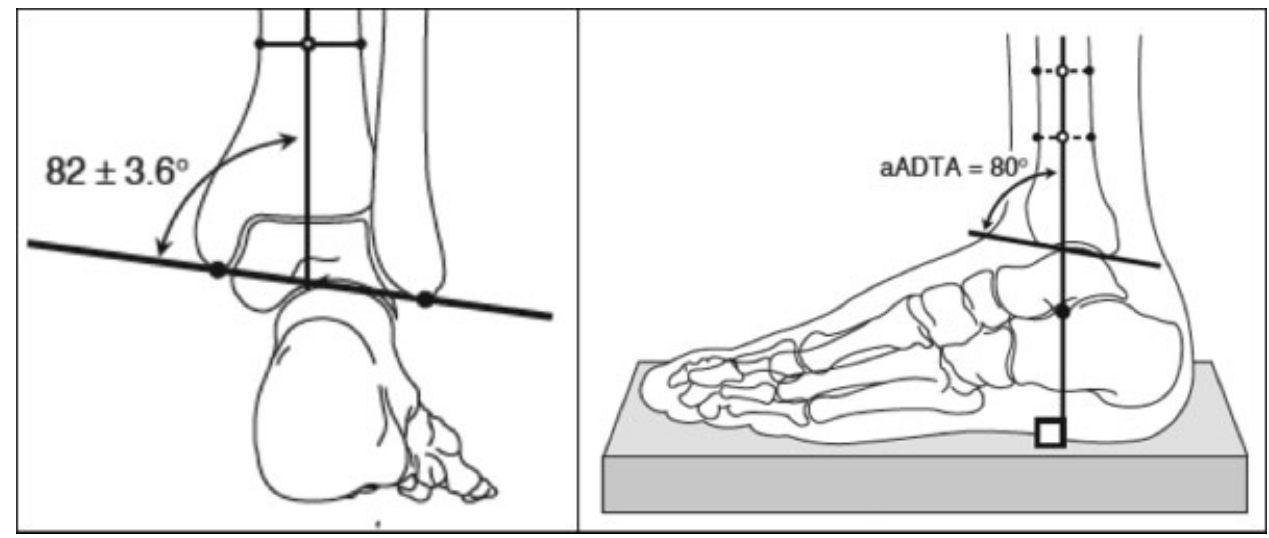

Fig. 3 Ángulo talocrual y ángulo tibial distal anterior. Lamm B, Paley D. Clin Podiatr Med Surg 21 (2004). 


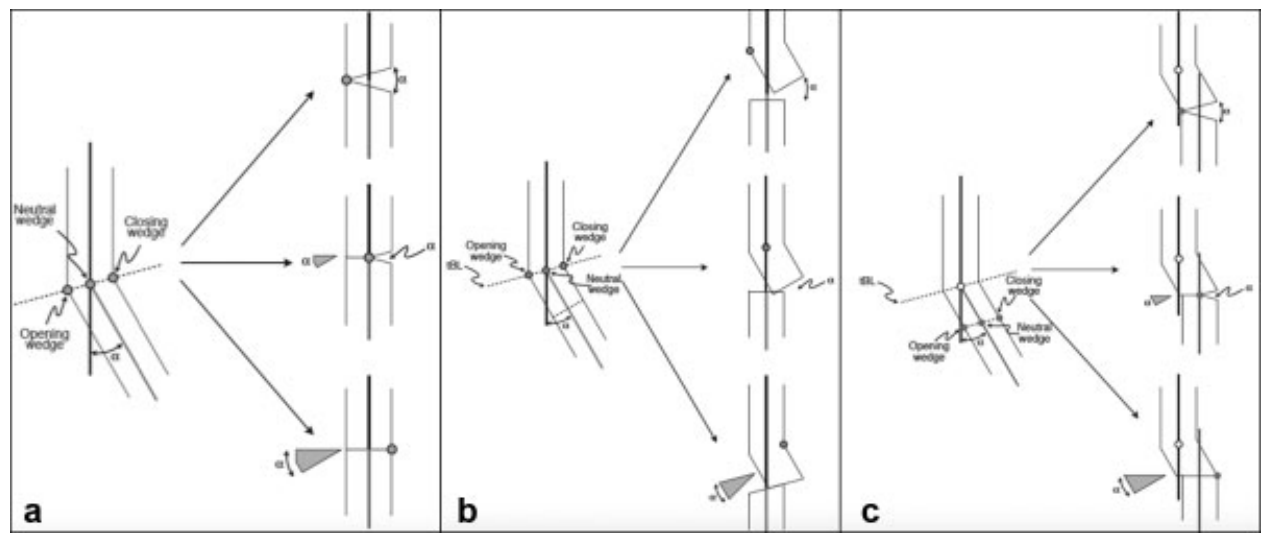

Fig. 4 Reglas de la osteotomía. (a) Regla y (b) Regla 2 y (c) Regla 3. Imagen extraída de Lamm B. Paley D. Deformity Correction planning for hindfoot, ankle, and lower limb. Clin Podiatr Med Surg 21 (2004).

Tercera regla: Si la bisagra y la osteotomía están a un nivel diferente del ápex, va a haber una deformidad de traslación (-Figura 4).

\section{Enfrentamiento Clínico}

\section{Historia y Examen Físico}

Para poder planificar adecuadamente un tratamiento, es de suma importancia obtener una historia detallada del paciente, la causa de la deformidad y los efectos que ésta tiene. Realizar un examen físico detallado es obligatorio, especialmente analizar la marcha y la fase estacionaria (bipedestación estática), ver si hay hiperlaxitud, contractura de partes blandas y discrepancias de extremidades. Además, se debe observar si hay mecanismos de compensación de las discrepancias: la pelvis puede inclinarse hacia abajo en el lado afectado, el tobillo de la extremidad corta se puede sostener en equino o la extremidad larga se puede flexionar en la rodilla, lo que permite que la rodilla de la extremidad corta se extienda completamente para poder estar plantígrado.

\section{Imágenes}

El set radiográfico básico es una telemetría de extremidades inferiores (que incluya pelvis, fémur, pierna, tobillo y pie) en proyección anteroposterior (AP) y lateral. Esas son importantes para evaluar el alineamiento general de las extremidades inferiores, ángulos de orientación de las articulaciones, discrepancias de longitud y el ápex de la deformidad. Esas proyecciones deben ser obtenidas incluso si la deformidad es solo del tobillo y pie. Además, se debe obtener radiografías con carga AP y lateral de tobillo y pie. Dado que la articulación subtalar no se visualiza bien en esas proyecciones, hay que agregar una proyección axial de calcáneo larga (Kleiger y Mankin) y Saltzman ${ }^{8}$ (- Figura 5).

Para evaluar la posición del pie durante la marcha, las radiografías deben ser obtenidas con el peso del paciente. Una radiografía de mortaja y lateral de tobillo es esencial para la medición y visualización precisa del plafón tibial, domo del talo y osteofitos en el tobillo anterior. Las radiografías con estrés también son importantes para diferenciar la contribución de partes blandas a la deformidad. Las radiografías en dorsiflexión máxima del tobillo ayudan a evaluar el equino versus el pinzamiento óseo anterior del tobillo. La fluoroscopía en tiempo real añade una cuarta dimensión a la evaluación del tobillo, la articulación subtalar y el movimiento del mediopie.

Otra imagen fundamental para el planeamiento quirúrgico es la tomografía computarizada (TAC) con carga del segmento afectado. Este nos permitirá reconocer deformidad en el plano axial y su magnitud, además de poder detectar causas secundarias de la deformidad (mal unión, no unión, infecciones, tumores). Lamentablemente, la TAC con carga no está disponible en nuestro medio.

\section{Planificación Quirúrgica}

Dependiendo del tipo de paciente y de la deformidad que tenga, vamos a definir el tratamiento a seguir. Algunos pacientes tienen deformidades leves, que no afectan la calidad de vida de manera significativa. A ellos se les podría plantear un manejo conservador, que va desde kinesiterapia con fortalecimiento muscular a órtesis a medida (plantillas entre otros). Muchos de esos casos logran llevar una vida adecuada, pero de todas formas no se está atacando el problema en sí: cuando hay una deformidad ósea que afecta el eje de la extremidad y que está ocasionando síntomas, la única forma de dar una solución definitiva es la cirugía. ${ }^{9}$

Antes de operar un paciente con deformidad de extremidad inferior es de suma importancia realizar una planificación quirúrgica ordenada, seguir los pasos que mostraremos y hacer un análisis global del paciente.

Primero que todo hay que detectar donde se encuentra el ápex de la deformidad (extremidad afectada, hueso afectado, si hay uno o más centros de angulación, en cuantos ejes está afectando). Luego de eso, haremos el análisis por planos. Importante es identificar si hay compensación de otras articulaciones como la subtalar y el medio pie, además de saber si ellas están rígidas o flexibles. Una vez finalizado eso, haremos las correcciones correspondientes. A continuación, se explicará el paso a paso de la corrección de deformidades. 


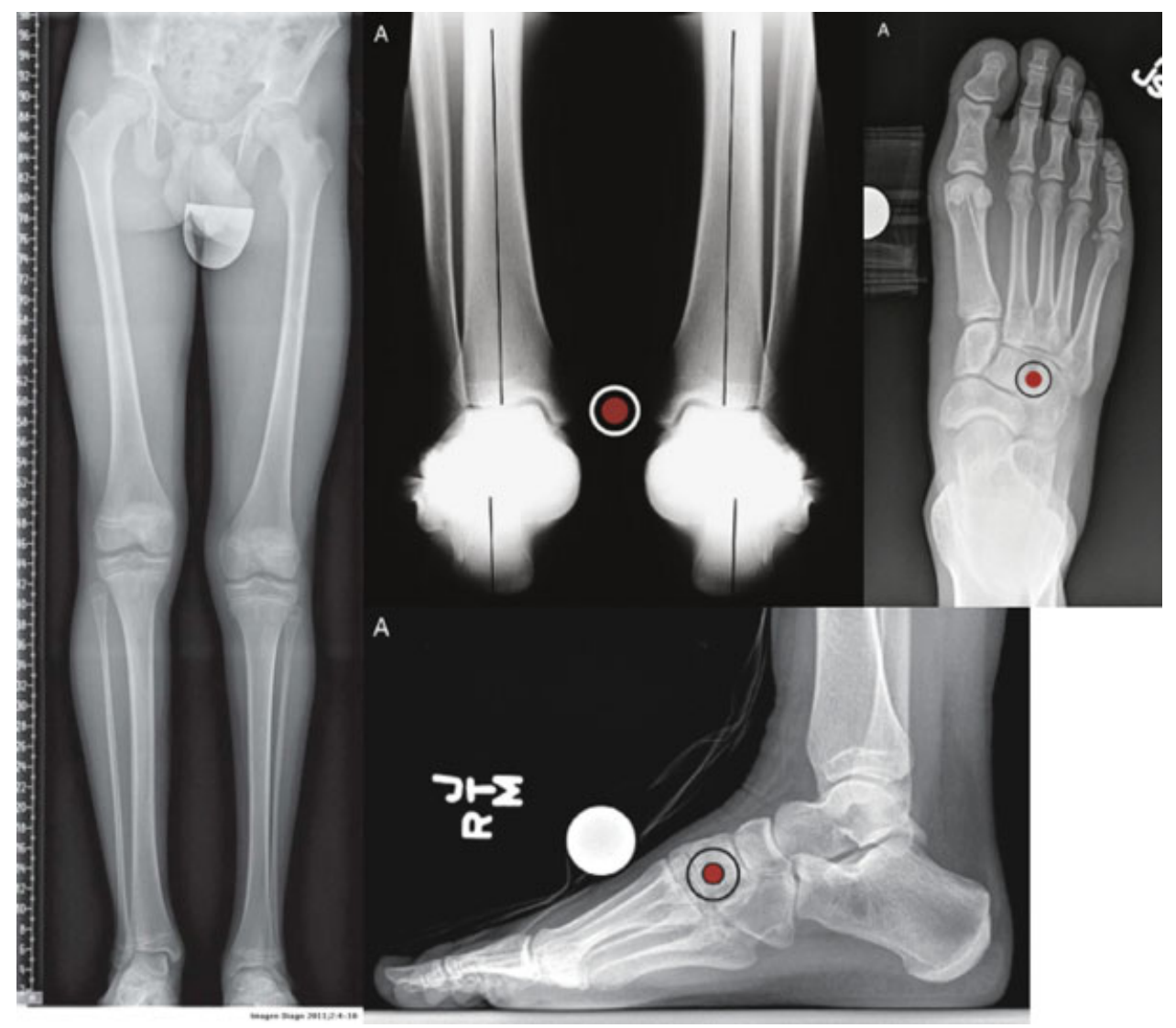

Fig. 5 Set radiográfico de estudio. Telemetría extremidades inferiores, AP y lateral de pie con carga, Kleiger.

\section{Análisis de Planos y Compensaciones Articulares en el Tobillo y Pie}

\section{Plano Frontal}

En la marcha normal, el vector de fuerza de reacción del suelo ("ground reaction force vector," GRFV) pasa lateral al tobillo e imparte un brazo palanca en valgo a la misma articulación, lo que crea una eversión de la subtalar.

Cuando hay deformidades en varo o valgo en la tibia, tienden a ser compensadas con la subtalar. Esa articulación tiene un rango de 15 grados de eversión y 30 de inversión, ${ }^{10}$ lo que permite una capacidad de corrección de deformidad tibial de la misma magnitud (el varo es peor tolerado). En caso que la deformidad sea mayor, comienza a compensar el medio pie, mediante pronación o supinación. Por esa razón, las deformidades de tobillo tienden a ser bien toleradas en la medida que la subtalar y medio pie sean flexibles. ${ }^{10}$

Cuando hay una deformidad en varo, la fuerza de reacción sobre el tobillo se medializa y tiende a ser muy sintomática por la poca compensación de la subtalar (pero sin generar artrosis significativa). Por otro lado, una deformidad en valgo es mejor compensada, pero tiende a dejar más cambios degenerativos.

Al planificar la corrección hay que considerar eso y la idea es tratar de restaurar el LDTA (ángulo distal tibial lateral) a $90^{\circ} .5$

\section{Plano Sagital}

El procurvatum y el retrocurvatum son compensados habitualmente por la flexión plantar o dorsal del tobillo. Un tobillo normal permite compensar con $20^{\circ}$ de dorsiflexión y $50^{\circ}$ de flexión plantar(por lo que el procurvatum es peor tolerado). ${ }^{5}$ En caso que la deformidad sea mayor que esa magnitud, el medio pie y subtalar ayudarán a compensar. Un procurvatum de tibia distal se diagnostica con un ADTA $>82^{\circ 6}$ y tiende a desplazar el retropié hacia posterior, disminuyendo el brazo de palanca del tobillo anterior. Un recurvatum de tibia distal se diagnostica con un ADTA $<78^{\circ 6}$ y tiende a dar síntomas más tardíos por la gran compensación de la flexión plantar.

Otra manera de evaluar la posición del tobillo es mediante el ángulo de inclinación del calcáneo, siendo $<13^{\circ}$ sugerente de un equino $\mathrm{y}>23^{\circ}$ de un arco aumentado. ${ }^{6}$

Para evaluar pie cavo y pie plano, se recomienda usar el ángulo de Meary.

\section{Compensaciones Rígidas}

Es imprescindible identificar cuando hay compensaciones rígidas. Una deformidad en varo de la tibia distal compensará con la subtalar en eversión y pronación del mediopie. Si eso se mantiene por años, evolucionará con rigidez. Si se realiza una cirugía de realineación tibial en este contexto, se deberán corregir también esas compensaciones para lograr un pie plantígrado, alineado y estable.

\section{Identificación del Ápex de Deformidad}

Para poder identificar el ápex de la deformidad hay que trazar el eje del hueso sobre y bajo la deformidad. Puede ser que haya 1 o más ápex de deformidad. Cada deformidad tiene que ser evaluada en el plano frontal, sagital y axial (perfil rotacional). 


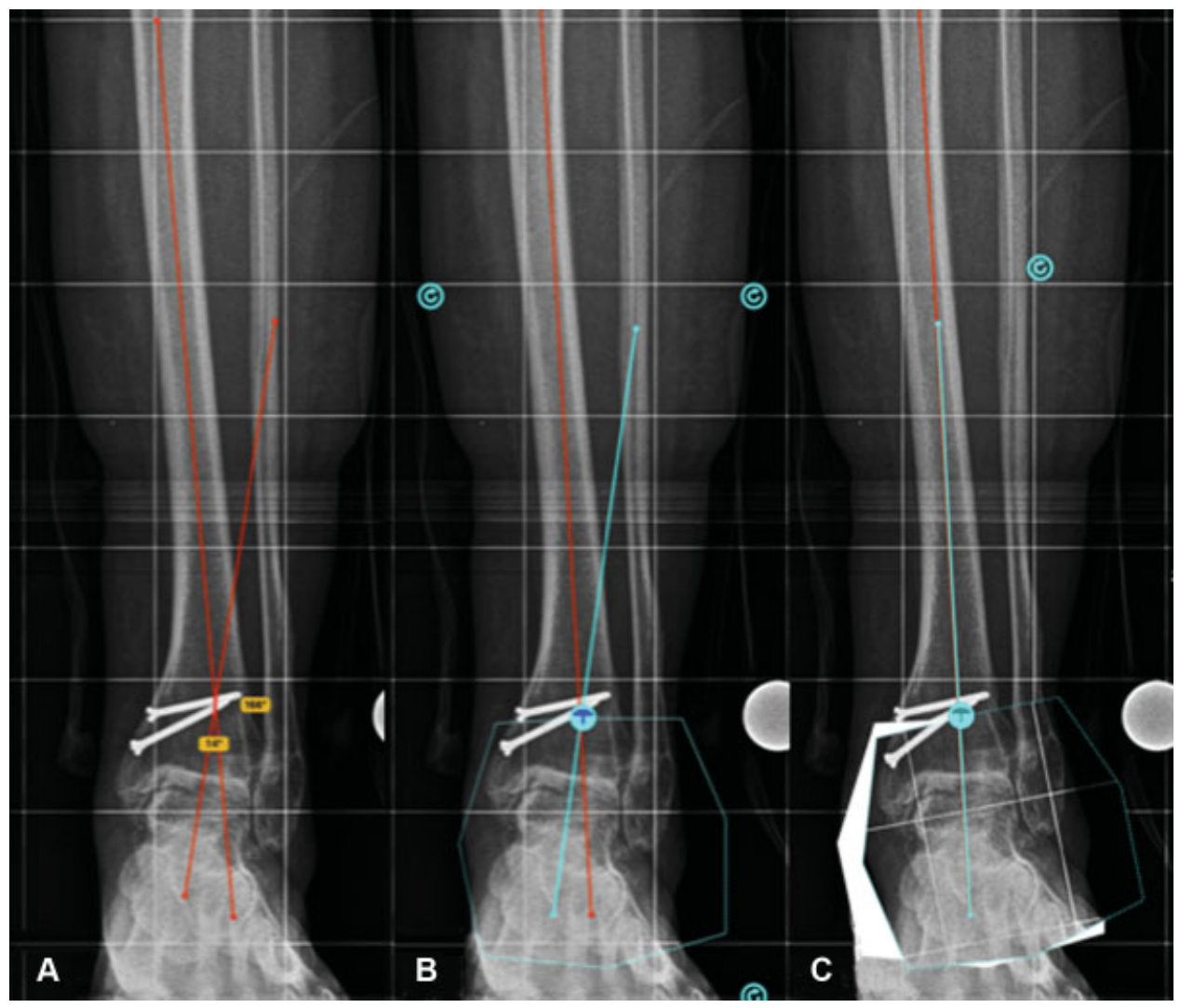

Fig. 6 (A) Ápex de deformidad en tibia; (B) Planificación ostetomía; (C) Post corrección.

\section{Para Deformidades en la Tibia Supramaleolares (- Figura 6) ${ }^{11}$}

Paso 1: Dibujar el eje diafisiario proximal y distal a la deformidad. Dibujar el eje mecánico del hueso.

Paso 2: Verificar que la rodilla y el tobillo no tengan alguna deformidad oculta. El MPTA normal (ángulo tibial medial proximal) es de $85-90^{\circ}$ y el LDTA (ángulo tibial distal lateral) es de $86-92^{\circ}$.

Paso 3: Una vez descartada una deformidad articular evidente, se intersecta línea diafisiaria proximal y distal. Ahí se encontrará el ápex de la deformidad. Medir la magnitud de la deformidad.

Paso 4: Marcar el sitio de osteotomía lo más cercano al ápex posible. El corte de la osteotomía debe pasar ese punto y bisectar el eje proximal y distal.

Paso 5: Realizar el corte hasta el centro del hueso; ese punto servirá como el hinge o bisagra descrito previamente.

Paso 6: Confirmar que el LDTA y MPTA se hayan normalizado posterior a la corrección.

Paso 7: Corregir la deformidad y fijarla mediante el uso de placa bloqueada.

Para Deformidades en el Tobillo Articulares (- Figura 7) Lo más frecuente son artrosis parciales, que tiene colapso de un área del Tobillo (Medial, Lateral, Anterior o Posterior). En esos casos, la corrección debe exagerarse para lograr una sobrecorrección de 3 a 5 grados

Paso 1: Dibujar el eje mecánico de la tibia
Paso 2: Dibujar la línea articular del tobillo y medir el LDTA.

Paso 3: Medir el ángulo plafond - maleolar.

Paso 4: Crear un LDTA normal de $89^{\circ}$ desde el centro de la articulación del tobillo.

Paso 5: Localizar el ápex de deformidad en la intersección de esas 2 líneas. Medir el ápex de deformidad.

Paso 6: Realizar la osteotomía $3 \mathrm{~cm}$ proximal a la articulación. Esa puede ser transversal (como la imagen) u oblicua en dirección a distal (llegar a $1 \mathrm{~cm}$ de la articulación del tobillo). Paso 7: Corregir la deformidad y fijarla mediante el uso de placa bloqueada.

\section{Para Deformidades en el Retropié (-Figura 8)}

Paso 1: Dibujar el eje mecánico de la tibia.

Paso 2: Dibujar la bisectriz del calcáneo. Primero dibujar los bordes medial y lateral del calcáneo. Luego usar esos bordes para encontrar la bisectriz.

Paso 3: Medir el ápex de deformidad intersectando esas 2 líneas. Ese ápex de deformidad no considera la relación de traslación lateral del eje del calcáneo en relación a la tibia que es de $1 \mathrm{~cm}$ aproximadamente (nota: el eje del calcáneo y la tibia son paralelos pero no coincidentes).

Paso 4: Para identificar el ápex de deformidad real considerando esta traslación, dibujar una línea punteada o de otro color que sea paralela a la bisectriz del calcáneo. El ápex real estará en la intersección de la línea diafisiaria tibial con la línea punteada. 


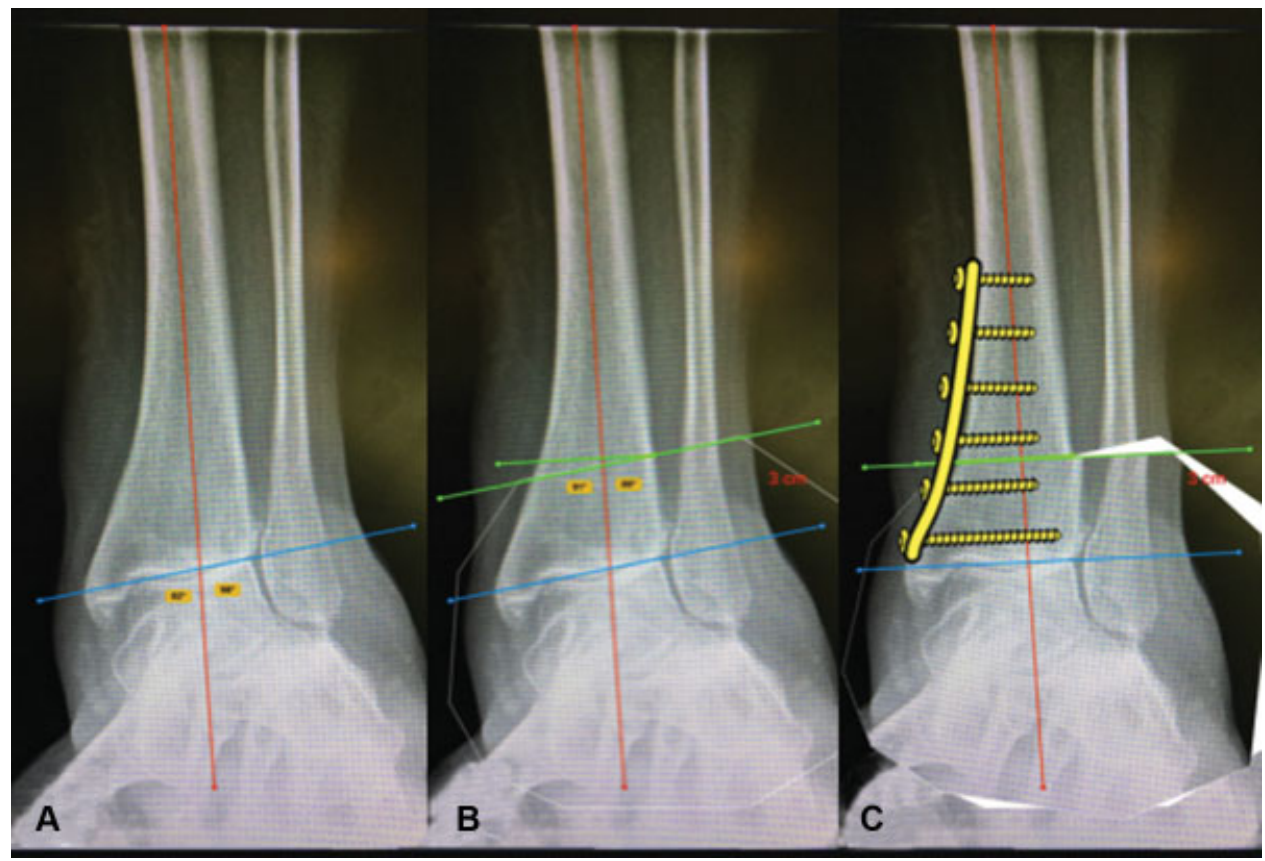

Fig. 7 (A) Ápex de deformidad en tobillo; (B) Planificación ostetomía; (C) Post corrección.

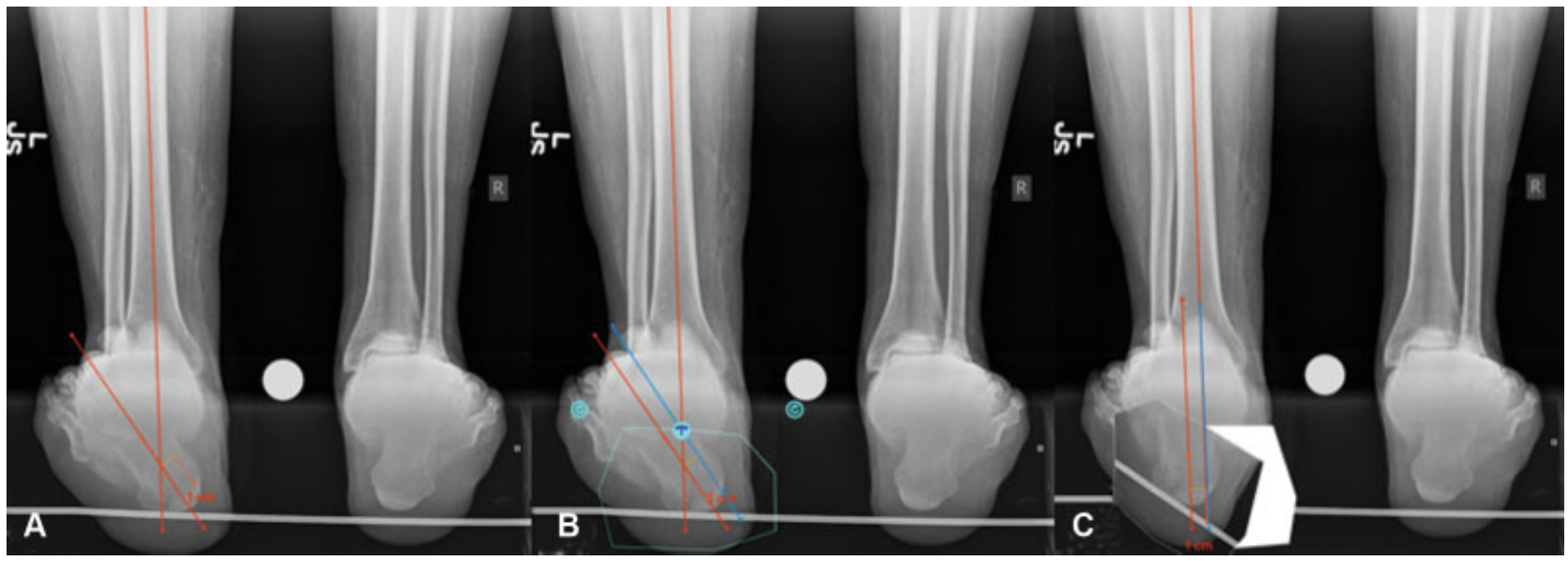

Fig. 8 (A) Ápex de deformidad en retropié de 35; (B) Bisectriz del calcáneo $1 \mathrm{~cm}$ a medial (azul) y punto de bisagra; (C) Post corrección.

Paso 5: Al hacer la osteotomía, se realiza una cuña de cierre lateral del ángulo medido.). Luego que se haya hecho la osteotomía y se remueva la cuña, trasladar el segmento distal de calcáneo $1 \mathrm{~cm}$ a lateral.

Paso 6: Fijar osteotomía con tornillos axiales compresivos sin cabeza.

\section{La Osteotomía}

Al momento de planificar la osteotomía, es importante recordar las reglas previamente descritas y el tipo de osteotomía a realizar. $^{11}$

Las diferentes osteotomías descritas son (-Figura 8):

- Osteotomía en cuña de cierre

- Osteotomía en cuña de apertura

- Osteotomía mixta
- Osteotomía en domo

- Osteotomía traslacional

\section{Conclusiones}

La corrección de deformidades en extremidades inferiores, especialmente en tobillo, retropié y pierna requieren de un gran conocimiento de los principios básicos y un adecuado plan quirúrgico. Es de vital importancia hacer una buena historia y examen físico detallado, además del set radiográfico para tener las herramientas esenciales antes de operar. Al momento de planificar la corrección es imprescindible evaluar la deformidad en su plano frontal, sagital y axial, además de la presencia de compensaciones que pueden ser flexibles o rígidas. Usar una planificación con cortes previamente calculados y considerar la extremidad como una unidad funcional logrará resultados más predecibles. 


\section{Conflicto de Intereses}

Los autores declaran no tener ningún conflicto de intereses.

\section{Bibliografía}

1 Butcher C, Atkins R. Principles of deformity correction. Curr Orthop 2003;17:418

2 Moreland JR, Bassett LW, Hanker GJ. Radiographic analysis of the axial alignment of the lower extremity. J Bone Joint Surg Am 1987;69(05):745-749

3 Paley D, Tetsworth K. Mechanical axis deviation of the lower limbs. Preoperative planning of multiapical frontal plane angular and bowing deformities of the femur and tibia. Clin Orthop Relat Res 1992;280(280):65-71

4 Bonnin M, Chambat P. [Current status of valgus angle, tibial head closing wedge osteotomy in media gonarthrosis]. Orthopade 2004;33(02):135-142
5 Standard SC, Herzenberg JE, Conway JD, Siddiqui NA. The Art of Limb Alignment. Sexta edición. Sinai Hospital of Baltimore 2017

6 Lamm BM, Stasko PA, Gesheff MG, Bhave A. Normal foot and ankle radiographic angles, measurements and reference points. J Foot Ankle Surg 2016;55(05):991-998

7 Paley D. Principles of deformity correction. Berlin: SpringerVerlag; 2002

8 Saltzman CL, el-Khoury GY. The hindfoot alignment view. Foot Ankle Int 1995;16(09):572-576

9 Paley D. Ankle malalignment. In: Kelikian AS, editor. Operative treatment of the foot and ankle. Stamford (CT): Appleton \& Lange; 1999:547-586

10 Chao EY, Neluheni EV, Hsu RW, Paley D. Biomechanics of malalignment. Orthop Clin North Am 1994;25(03):379-386

11 Paley D, Herzenberg JE, Tetsworth K, McKie J, Bhave A. Deformity planning for frontal and sagittal plane corrective osteotomies. Orthop Clin North Am 1994;25(03):425-465 